Document downloaded from:

http://hdl.handle.net/10251/103506

This paper must be cited as:

Antonino-Daviu, J.; Quijano Lopez, A.; Climente Alarcon, V.; Garín-Abellán, C. (2017). Reliable Detection of Rotor Winding Asymmetries in Wound Rotor Induction Motors via Integral Current Analysis. IEEE Transactions on Industry Applications. 53(3):2040-2048. doi:10.1109/TIA.2017.2672524

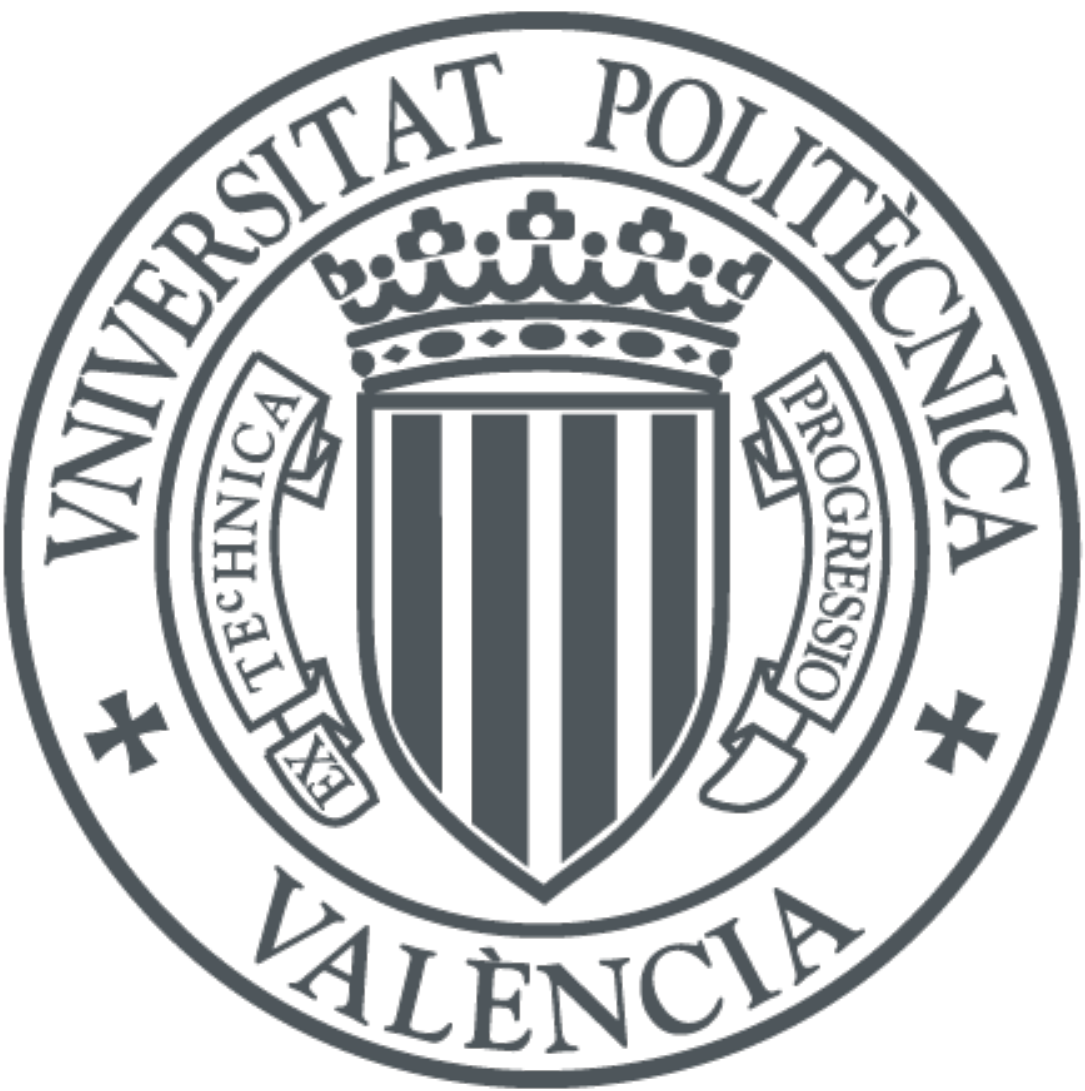

The final publication is available at

http://doi.org/10.1109/TIA.2017.2672524

Copyright Institute of Electrical and Electronics Engineers

Additional Information 


\section{Reliable Detection of Rotor Winding Asymmetries in Wound Rotor Induction Motors via Integral Current Analysis}

\author{
Jose Antonino-Daviu, Alfredo \\ Quijano-López \\ Instituto Tecnologico de la Energia, \\ Universitat Politècnica de València, \\ Camino de Vera s/n, 46022, \\ Valencia, SPAIN \\ joanda@die.upv.es
}

\author{
Vicente Climente-Alarcon \\ Department of Electrical \\ Engineering and Automation, \\ Aalto University, P. O. Box 13000, \\ FI-00076 Aalto, FINLAND \\ viclial@ieee.org
}

\author{
Carlos Garín-Abellán \\ FYM-Italcementi Group \\ Ctra. Almería, km.8, \\ 29720 Málaga, SPAIN \\ c.garin@fym.es
}

\begin{abstract}
Current analysis has been widely employed in academy and industry for the diagnosis of rotor damages in cage induction motors. The conventional approach based on the FFT analysis of steady-state current (MCSA) has been recently complemented with the development of alternative techniques that rely on the time-frequency analysis of transient quantities of the machine. These techniques may bring important advantages that are related to the avoidance of eventual false indications provided by the classical MCSA. Moreover, their application is also suitable for variable speed conditions. However, the application of current-based methodologies to wound rotor induction motors (WRIM) has been much less studied and, hence, their validation in field WRIM is scarce. The present work proposes the application of an integral methodology based on the analysis of both stationary and transient currents for the diagnosis of winding asymmetries in WRIM. The method, based on up to five different fault evidences, is validated in laboratory motors and it is subsequently applied to a large field motor $(1,500$ $\mathrm{kW}$ ) that was showing signs of abnormal rotor functioning. The results prove that the method is of interest for the field since it helps to ratify without ambiguity the existence of eventual asymmetries in the rotor windings, with no interference with the machine operation. However, due to the complex constructive nature of the rotor winding as well as the presence of auxiliary systems (slip rings, brushes, contactors, etc...), once the fault presence is detected, it may be interesting the utilization of complementary tools to accurately locate the root cause of the asymmetry.
\end{abstract}

Keywords-induction motors; wound rotor; fault diagnosis; transient analysis; wavelet transforms

\section{INTRODUCTION}

Monitoring electrical quantities is an interesting option for the determination of the health of induction motors (IM). In comparison with other quantities, it brings important benefits as remote monitoring capability, low implementation cost and non-invasive nature, amongst other [1-3]. More specifically, motor current monitoring is the best option to diagnose certain motor failures, such as eventual damages in the rotor cage. There are many works in the literature that have deepened in the application of current-based techniques to detect such failures in cage induction motors; the early works, published several decades ago, proposed the Fourier analysis of the steady-state current to assess the amplitude of different components amplified by the rotor fault [4-6]. This is the basic idea of the classical Motor Current Signature Analysis (MCSA) that has a widespread use in industry to detect rotor failures and is the technique implemented in the few available commercial devices for monitoring the rotor health condition. Recently, an alternative diagnostic trend based on analysis of transient currents (e.g. motor startup current) has been proposed. This new approach was conceived to avoid some drawbacks of MCSA, such as its eventual false diagnostic indications (either false positives or negatives) that may rise for certain motor constructive characteristics (presence of rotor cooling ducts, motors with double cage rotors, anisotropic rotor cores...) or operating conditions (load torque oscillations, no-load conditions, ...) as well as its unsuitable application under varying speed conditions [7-16].

In spite of the vast amount of works devoted to the application of currents analysis to diagnose rotor faults in induction motors, the vast majority is focused on cage induction machines. With regards to WRIM, several works in the literature have proposed techniques relying on the analysis of vibrations [29-30] or other quantities [31-32] for the detection of stator and rotor winding faults. Few works have dealt with the application of current based techniques (especially, transient-based) to detect rotor failures in WRIM [17-19, 24, 33-35] and even fewer have validated this application in real industrial machines, where the presence of such fault was reported. Note, however, that although WRIM are not so common as cage IM, they have some important advantages, such as their ability to develop high starting torques at lower startup currents (by inserting external resistors in series with the rotor winding) [20]. The use of WRIM is rather common in large power applications (typically, above $1,000 \mathrm{hp}$ ). These large motors are usually expensive, critical and with difficult repair and, hence, a monitoring system that can detect and diagnose incipient faults is of paramount importance. Common industrial applications are, among other, ball and sag mills, cranes, pumps, fans and blowers, conveyors, 
chippers or hoists [20-21]. It is well known that, operating as generators, wound rotor induction machines are extensively employed in areas such as wind power generation and, in this field, many works have investigated the diagnosis of different failures in double fed induction generators [36-38].

The present work is focused on the diagnosis of rotor winding asymmetries in WRIMs by means of currents analysis [27]. The underlying idea is to complement several indicators based on the classical MCSA with additional evidences obtained from the advanced stator startup current analysis as well as, when available, from measured rotor currents. It must be said that, although the possibility of rotor currents measurement exists for most of these machines (unlike what happens in cage motors), in many cases the access to the rotor currents is not so easy and, even when accessible, its measurement cannot often be carried out under safe and simple conditions.

The proposed method relies on a total number of four fault evidences that are obtained from the simple measurement of a single stator phase current during the startup and a portion of the steady-state. A complementary fifth indicator is also proposed based on the measured rotor currents, if available. Laboratory data are employed to initially validate the methodology. Afterwards, it is tested in a field WRIM operating in a cement plant that was showing symptoms of abnormal operation. The application of the approach to this machine confirmed the presence of a significant rotor asymmetry with potential severe consequences for the machine; once the rotor winding asymmetry was diagnosed, a subsequent infrared inspection of the rotor enabled to determine the exact point where the asymmetry was originated.

\section{INTEGRAL METHOD BASED ON CURRENT ANALYSIS}

The method proposed in this work for the robust detection of rotor asymmetries in WRIM is based on different fault evidences rising from the application of rotor assessment techniques that combine the analysis of both steady-state and startup currents [17, 24]. On the one hand, two of these evidences are derived from the classical MCSA of the stator current. Reference [25] applied the Fortescue's theorem to the unbalanced three-phase system conformed by the rotor currents in a WRIM with a rotor winding asymmetry; it was shown there that the presence of the asymmetry leads to the amplification of certain harmonic families, with diverse origin (magnetic or mechanical), in the steady-state current spectrum [25]. As a consequence, the two first evidences of the methodology proposed here are derived from the evaluation of two of these harmonic families.

On the one hand, the first evidence comes from the evaluation of the amplitudes of the family of fault components given by (1) ( $f=$ supply frequency, $s=$ slip, $k=1,2,3 \ldots)$ [4, 17, 25]. This family yields a set of sidebands around the supply frequency component (the higher the value of $k$, the lower their amplitude). More specifically, for $k=1$, the main sideband components are obtained; in cage IM, an amplitude of the lower sideband (LS) ( $k=1$; negative sign) between $\sim-55 \mathrm{~dB}-45 \mathrm{~dB}$ relative to the fundamental is typically used as an alarm threshold that informs on a certain level of rotor damage. These thresholds are no longer valid for WRIM due to the different constructive characteristics of the rotor winding for such machines.

$$
f_{\text {faml }}=f \cdot(1 \pm 2 \cdot k \cdot s)
$$

The second fault evidence relies on the evaluation of the amplitudes of a second family of harmonics amplified by the rotor fault; these harmonics are given by expression (2), as shown in [25]. For $k=1$, this expression yields two harmonics that are located near the fifth winding harmonic and with frequencies given by: $f \cdot(5-4 \cdot s)$ and $f \cdot(5-6 \cdot s)$. The evaluation of these two harmonics is the second evidence of the methodology. Note that, as commented in some works [22], these harmonics can be a valuable informational source to discriminate between rotor damages and other effects, such as the presence of load oscillations, since these harmonics are less affected by pulsations in the driven load torque (that have a much lower influence on the fault components at higher frequencies) [22].

$$
\begin{gathered}
f_{\text {fam } 21}=f \cdot[(6 \cdot k-1)-6 \cdot k \cdot s] \\
f_{\text {fam } 22}=f \cdot[(6 \cdot k-1)-(6 \cdot k-2) \cdot s]
\end{gathered}
$$

It must be remarked that the previous harmonic families are also amplified in the case of cage induction motors and are even used in some commercial devices for the rotor cage assessment in such motors. Nonetheless, in past works, it was proven that the two previous evidences were not enough to confirm the presence of rotor failures in cage induction motors; this was due to the fact that there are some situations where other harmonics that are not related to the fault can be misinterpreted as fault components; these situations can lead to false positive indications [7-10, 14-15]. In other cases, although the fault is present, the amplitudes of the previous components may be low or they may not be easily assessable, a fact that can lead to eventual false negative indications [8, $11,13]$. Some of these situations leading to eventual false indications in cage motors can be extrapolated to WRIM, as the presence of load torque oscillations (that can lead to false positive indications, if only the sidebands given by (1) are considered) or the diagnosis under no-load conditions (false negative indications).

In order to prevent the occurrence of false indications, two additional evidences based on the analysis of the startup current via Continuous and Discrete time-frequency transforms are proposed in the present work [23]. The third evidence results from the STFT analysis of the startup current and is based on the detection of the $\mathrm{V}$-shaped pattern caused by the evolution of the lower sideband component (if present) in the time-frequency map resulting from the application of this transform; this pattern was well characterized in previous works [23-24] and its identification is a reliable indicator of the existence of the rotor winding asymmetry [24]. For instance, some works propose the startup analysis and the 


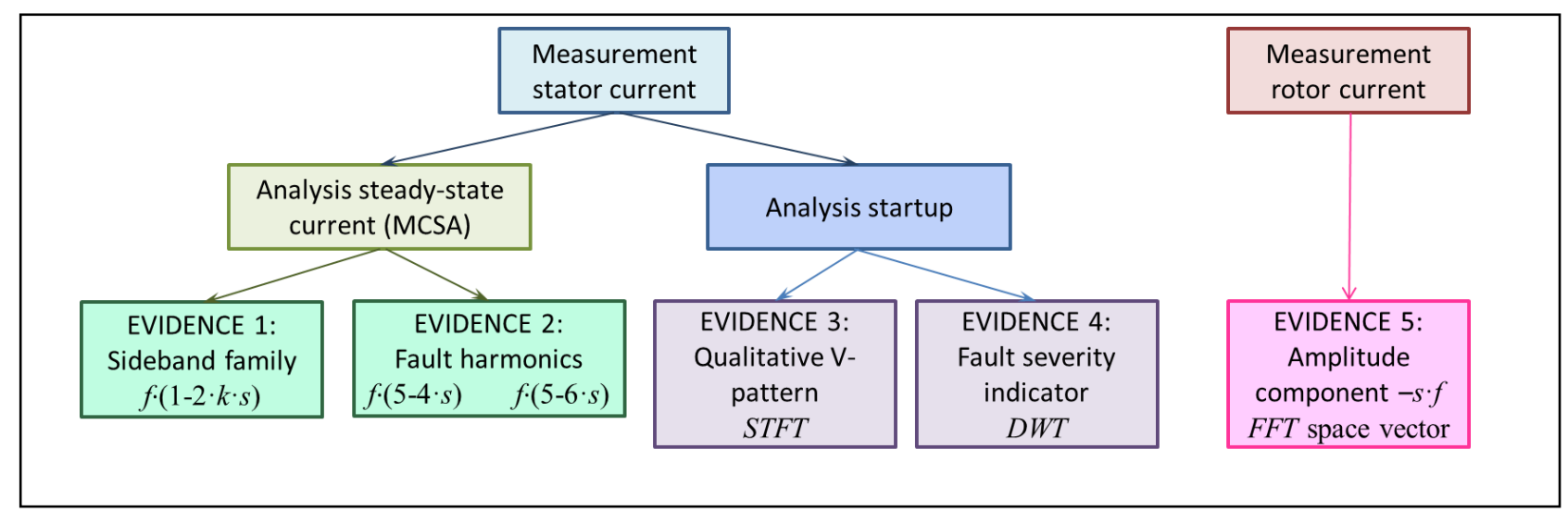

Fig. 1. Schematic representation of the proposed method for diagnosis of rotor asymmetries in WRIM.

identification of this pattern to discriminate between load effects and rotor damages in cage motors $[8,28]$. On the other hand, the fourth evidence is based on the computation of the fault severity indicator based on the DWT of the startup current $[12,15]$. This indicator $\left(\gamma_{D W T}\right)$ is given by expression (3) and relates the energy of the total current signal (i) with that of the wavelet signal containing most of the fault component evolution $(d)$. A value below $50 \mathrm{~dB}$ is indicative of a certain level of rotor asymmetry in cage IM.

$$
\gamma_{D W T}(d B)=10 \cdot \log \left[\frac{\sum_{j=N b}^{N s} i_{j}^{2}}{\sum_{j=N b}^{N s}\left[d_{n f+1}(j)\right]^{2}}\right]
$$

Finally, in those industrial applications where the three rotor currents can be accessed and measured, a fifth indicator is defined; it relies on the evaluation of the component at $-s \cdot f$ in the rotors-currents space vector spectrum. This component was well characterized in [4]. The schematic representation of the proposed integral current-based diagnosis approach for WRIM is illustrated in Fig.1.

\section{APPLICATION TO LABORATORY AND FIELD MOTORS}

The proposed method was validated first in the laboratory on a $4 \mathrm{P}, 400 \mathrm{~V}, 11 \mathrm{~kW}$ WRIM where different levels of rotor winding asymmetry were forced (Fig.2). The rotor windings were accessible through terminals which enabled their connection with external resistors. The rotor asymmetry was controlled using an external rheostat inserted in series with one of the rotor windings. By varying the rheostat resistance, up to ten different asymmetry levels were simulated. Stator and rotor currents were captured both during the startup and during steady-state. The sampling rate used for capturing the signals was $5 \mathrm{kHz}$ and the total register time was 100 seconds. Afterwards, the method was applied to a field $12 \mathrm{P}, 3.3 \mathrm{kV}$, $1,500 \mathrm{~kW}$ WRIM that was driving a ball mill in a cement plant (Fig. 3) (the data acquisition parameters were similar those used in the laboratory motors). Preliminary MCSA analysis carried out by engineers of the plant had revealed some symptoms of an abnormal condition of the rotor winding, so it was decided to apply the five-step methodology proposed in this work to determine the real condition of the winding.

The application of the method to laboratory motors is depicted in Figs. 4 to 7 . Only three levels of rotor asymmetry are shown in the graphs: high $\left(\mathrm{R}_{\text {added }}=1\right.$ ' $8 * \mathrm{Rr}$ with $\mathrm{Rr}=$ rotor phase resistance), medium $\left(\mathrm{R}_{\mathrm{added}}=0{ }^{\prime} 7 * \mathrm{Rr}\right)$ and healthy motor. Figs. 4 and 5 show the MCSA that was employed to obtain the two first fault evidences (high asymmetry depicted in blue color, medium in red and healthy in black). In these figures, the load level of the machine was $40 \%$ of the rated load.

On the first hand, Fig. 4 shows how the family $f \cdot(1 \pm 2 \cdot k \cdot s)$ clearly appears for the motors with asymmetry (the higher the asymmetry, the greater the amplitudes). In the healthy motor they also appear but with much lower amplitudes (note that, unlike cage IM, in WRIM there are a lot of elements that may lead to a certain rotor winding asymmetry (slip rings, brushes, coils connections, external contacts...), a fact that may lead to observable amplitudes of fault harmonics even if the machine is not considered as faulty).

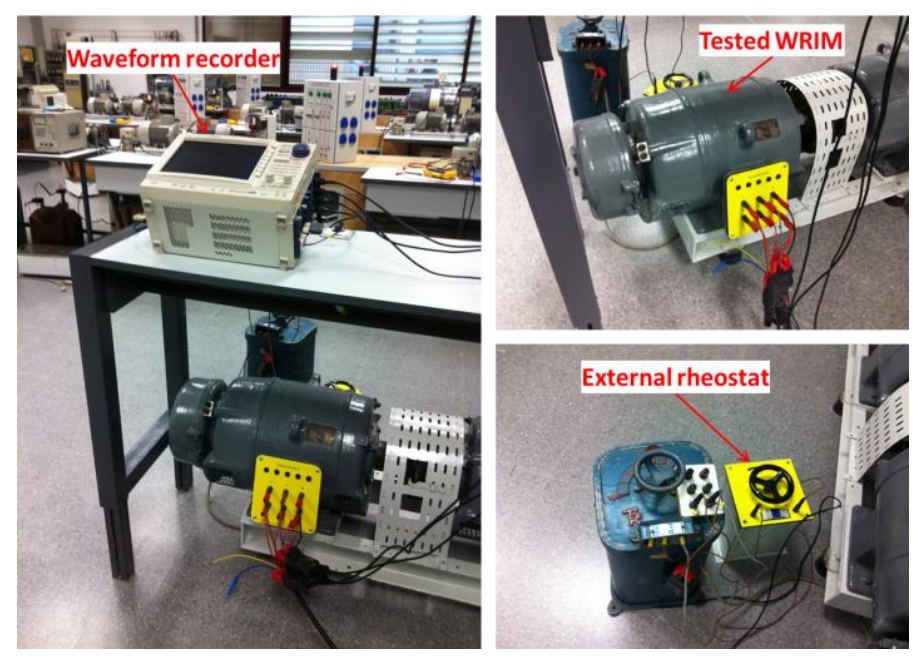

Fig. 2 Laboratory 4P, 400V, 11kW WRIM and external rheostat to force the asymmetry 

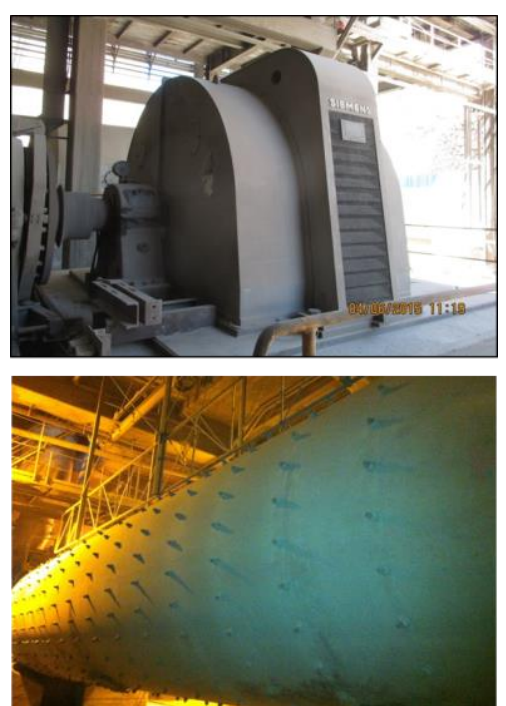

Fig. 3 Photographs of the field WRIM (12P, $3.3 \mathrm{kV}, 1,500 \mathrm{~kW})$ and of the driven mill.

On the other hand, Fig. 5 shows the clear presence of the fault harmonics near the fifth winding harmonic; these harmonics, located at $f \cdot(5-6 \cdot s)$ and $f \cdot(5-4 \cdot s)$ had higher amplitudes for the machines with asymmetry (second evidence to diagnose the presence of the failure).

To ratify the conclusions obtained from the analysis of the MCSA evidences, the startup current analysis is carried out, via Continuous and Discrete time-frequency transforms [23], following the methodology illustrated in Fig.1. Fig. 6 shows the STFT analyses of the startup current for the three considered cases. The foundations of this signal processing tool are well-known [26] and it can be programmed in conventional mathematical software packages. Note the clear presence of the V-pattern caused by the LS in the startup current $[8,23]$ for the faulty cases; in healthy condition, the pattern is much less evident since the LS has lower amplitude.
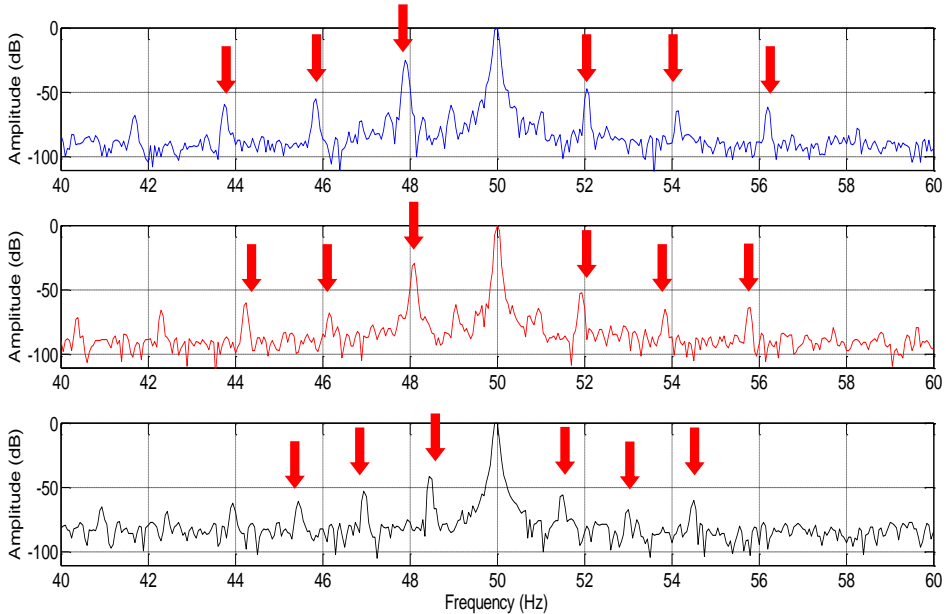

Fig. 4 EVIDENCE 1: Presence of the family $f \cdot(1 \pm 2 \cdot k \cdot s)$ in MCSA. High asymmetry (blue color),medium (red color) and low asymmetry (black).
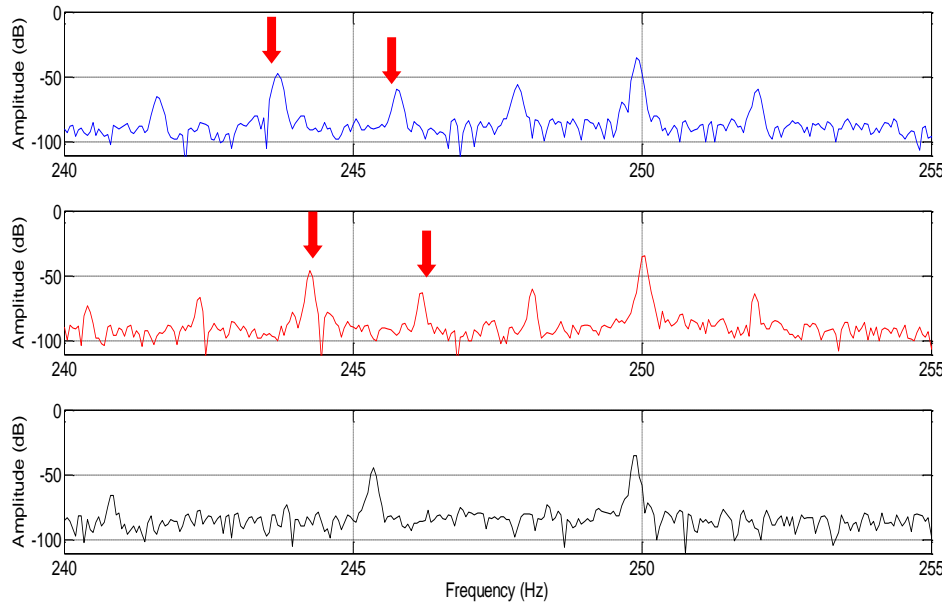

Fig. 5 EVIDENCE 2: Presence of the harmonics $f \cdot(5-4 \cdot s)$ and $f \cdot(5-6 \cdot s)$ in MCSA. High asymmetry (blue color),medium (red color) and low asymmetry (black).

This is ratified when computing the quantification indicator based on the DWT [12, 15] (evidence 4); Table I shows the values of the indicator $\gamma_{D W T}$ for different cases tested. Note the value of the fault indicator sensibly decreases when the level of asymmetry increases showing variations of more than 20 $\mathrm{dB}$ between healthy condition and medium asymmetry level.

Finally, Fig. 7 shows the rotor-currents space-vector FFT spectra for the three previous experimental cases. Note how, in the case that these currents can be measured (this can depend on many issues as rotor windings accessibility, etc...), the FFT analysis of their space vector can provide very useful information: as observed, for the machines with asymmetry, the component at $-s \cdot f$ has a prominent amplitude (more than $15 \mathrm{~dB}$ versus healthy condition). Hence, this component is amplified by the rotor asymmetry in the rotor-currents space vector spectrum (as justified in [17]). This evidence, together with the previous ones, enables to reliably diagnose the presence of the fault.

TABLE I.

EVIDENCE 4: Fault indicator based on DWT $\left(\gamma_{D W T}\right)$

\begin{tabular}{|c|c|}
\hline Inserted R & Indicator $\left(\boldsymbol{\gamma}_{\boldsymbol{D W T}}\right) \quad(\mathbf{d B})$ \\
\hline $1^{\prime} 8^{*} \mathrm{Rr}$ & 12,7 \\
$0^{\prime} 7^{*} \mathrm{Rr}$ & 18,2 \\
$0^{\prime} 1^{*} \mathrm{Rr}$ & 36,5 \\
\hline 0 (healthy) & 43,2 \\
\hline
\end{tabular}




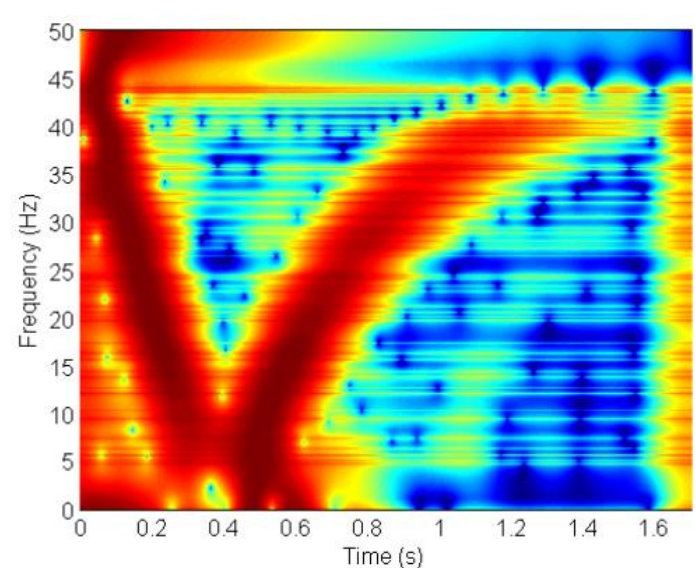

(a)

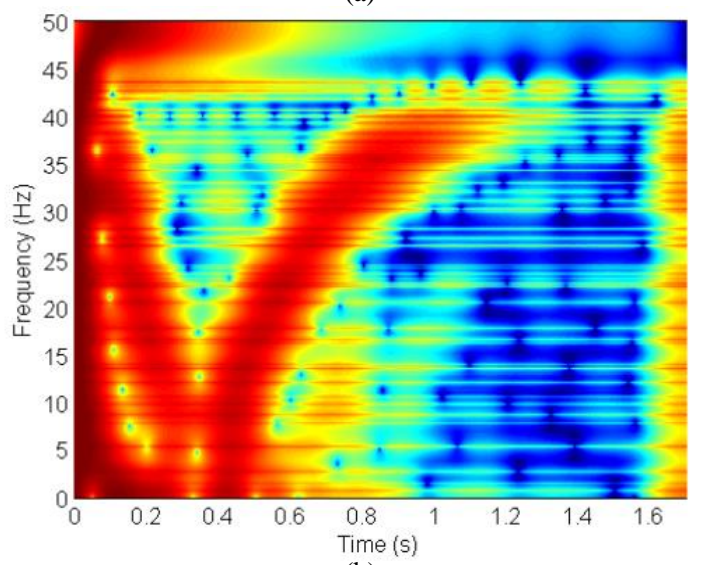

(b)

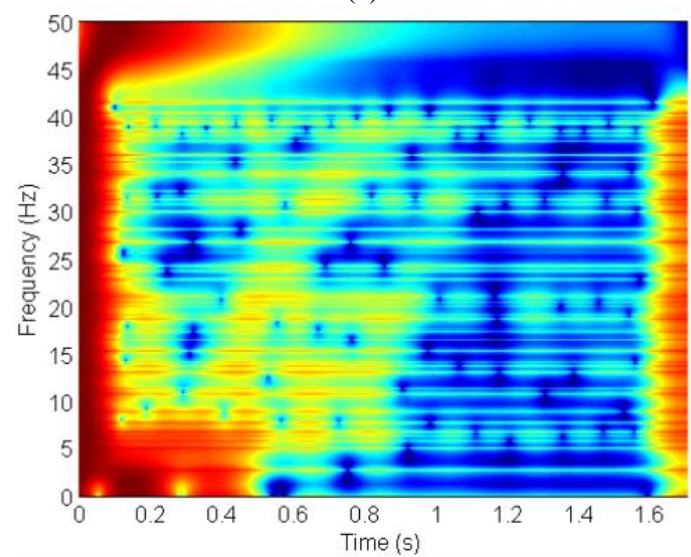

(c)

Fig. 6 EVIDENCE 3: STFT of the startup current of WRIM for a) high rotor asymmetry, b) medium asymmetry and c) healthy.
The proposed methodology, based on five different evidences, obtained from the analysis of stator currents (steady-state and startup) and rotor currents (if measured) was afterwards validated in a field WRIM. This was a $1,500 \mathrm{~kW}$ motor that was driving a ball mill in a cement factory (see Fig. 3). An unexpected fault in this motor could have very significant consequences for the cement company since a forced outage could mean costs above $\$ 50,000$.

Preliminary MCSA analyses carried out by the plant engineers were showing symptoms of rotor anomaly so that the method proposed in the present paper was applied to determine whether the fault was present or not. Figs. 8 and 9 show the application of the first step of the methodology (MCSA) to obtain the fault evidences 1 and 2; note the presence of the fault components in both Figures (both the family $f \cdot(1 \pm 2 \cdot k \cdot s)$ in Fig. 8 and the components $f \cdot(5-4 \cdot s)$ and $f \cdot(5-6 \cdot s)$ in Fig. 9). The prominent amplitudes of these components seem to confirm the presence of the fault (the LS has an amplitude of around $-33 \mathrm{~dB}$ ).

Fig. 10 depicts the STFT analysis of the stator startup current for the field WRIM. The presence of the V-shaped pattern caused by the evolution of the LSH is clearly observed (Evidence 3). On the other hand, the computation of the fault indicator based on the DWT $\left(\gamma_{D W T}\right)$ yields a value of $25 \mathrm{~dB}$ (much lower than the threshold used in cage motors- $50 \mathrm{~dB}$ and similar to the values for medium-high asymmetry in lab motors) confirming the presence of the rotor asymmetry (Evidence 4). Finally, Fig. 11 reveals the significant amplitude of the component at $-s \cdot f$ in the rotor-currents space-vector spectrum (Evidence 5).

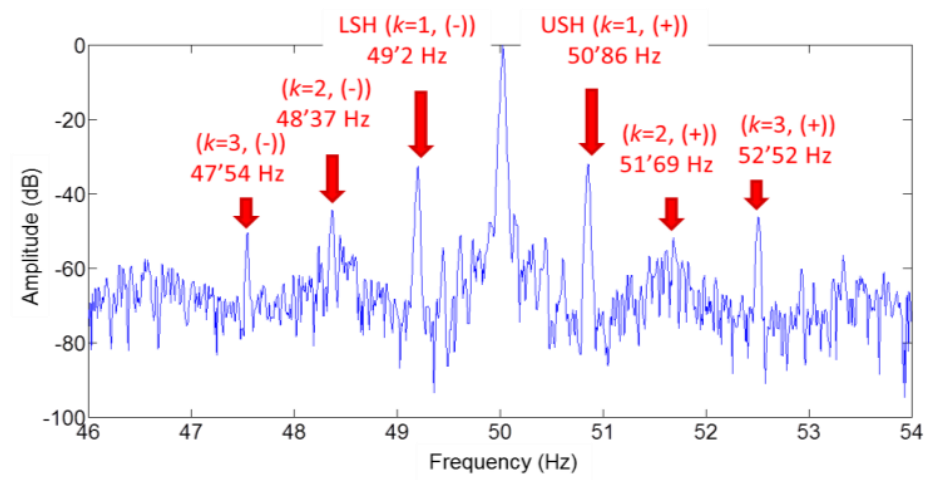

Fig. 8 Field motor - EVIDENCE 1: Presence of the family $f \cdot(1 \pm 2 \cdot k \cdot s)$ in MCSA

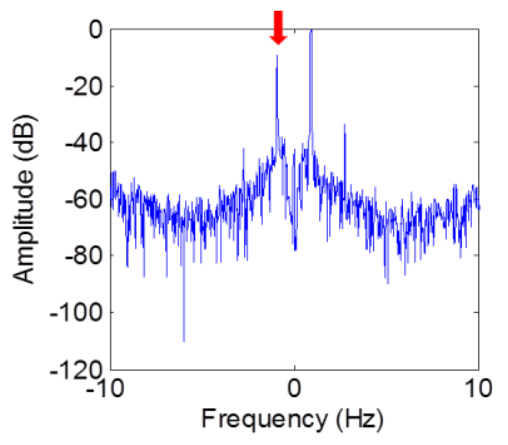

(a)

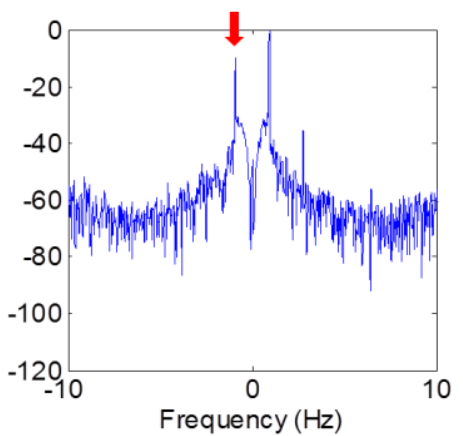

(b)

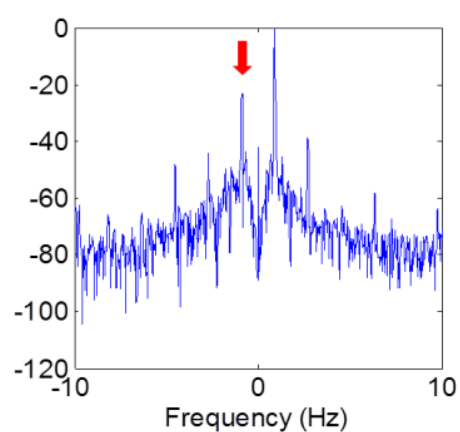

(c)

Fig. 7 EVIDENCE 5: Rotor-currents space-vector spectrum for the WRIM with a) high rotor asymmetry, b) medium and c) healthy. 


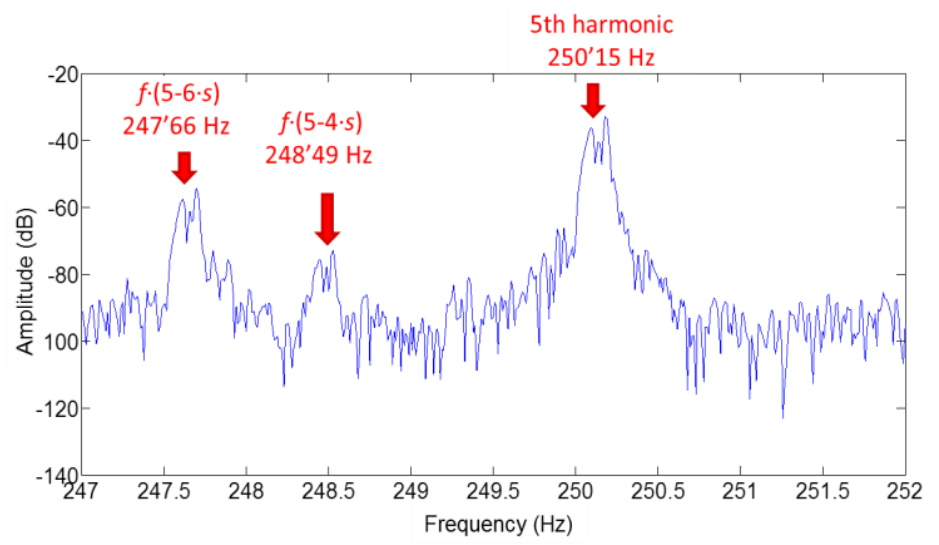

Fig. 9 Field motor - EVIDENCE 2: Presence of the harmonics $f \cdot(5-4 \cdot s)$ and $f \cdot(5-6 \cdot s)$ in MCSA.

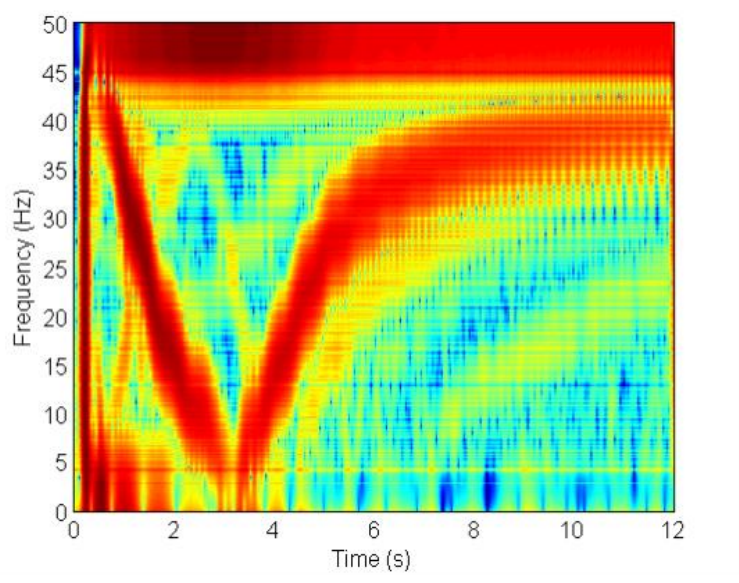

Fig. 10 Field motor - EVIDENCE 3: STFT of the startup current.

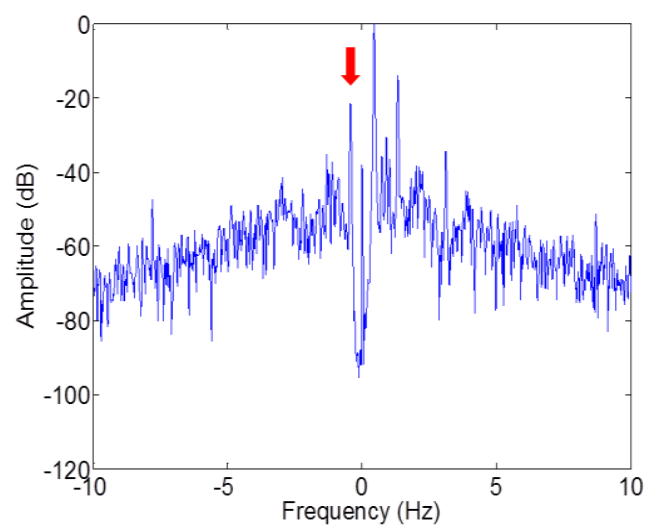

Fig. 11 Field motor- EVIDENCE 5: Rotor-currents space-vector spectrum .

Note that four different factory workers were needed to open the enclosure that enabled the access to the rotor winding and that the measurement of these currents required the adoption of especial safety measures; this is illustrative on the fact that measuring rotor currents is not always easy and safe in field WRIM and, hence, that the diagnosis of the rotor winding condition should not only rely on them.
In conclusion, all five fault evidences were indicating the presence of a significant asymmetry in the rotor winding of the field WRIM.

Once the asymmetry presence had been confirmed with the proposed methodology, the problem was to determine the exact point where the asymmetry was originated. In this sense, the rotor winding of WRIM is not as simple as the cage of a cage IM under a constructive point of view and there were any parts in the winding where the asymmetry source could be localized. The employed procedure was to analyze each possibility, discarding one by one the incorrect options: in this regard, the starting rheostat was discarded as potential source of the asymmetry since it was short-circuited after the startup and, as it was shown in the analysis, the asymmetry was detected both during the startup and during steady-state; this fact shows another interesting advantage of the proposed method since it enables to discard possible causes of the rotor asymmetry depending on the evidences that rise from the results (startup and/or steady-state).

Other possible source was an eventual defective connection in the contactor that enabled the connection of the rotor winding to the rheostat and the subsequent short-circuit of the winding at steady-state. A thermographic inspection of the contactor showed not abnormal temperatures and no evidences of defects, high-resistance points or other anomalies. Finally, the potential causes of the asymmetry were reduced to two options: problems in the slip rings/brushes system or internal problems in the winding (cracked turns, defective connections between coils, different sizes of coil conductors, etc...). This last option was more problematic since it may imply to adopt the drastic decision of motor rewinding with subsequent costs (higher than \$20,000).

To ratify that the problem was in one of these parts, the rotor winding resistance test was carried out; a digital microhmeter was used to measure the resistive unbalance between the rotor phases (see Fig. 12). The test revealed a delta $\mathrm{R}$ parameter of near $6 \%$. The measured resistance included the resistance of the rotor winding as well as of the slip ring/brushes system; the detected unbalance confirmed that the problem was located in one of this two elements.

Finally, a thermographic inspection of the slip ring/brushes system was carried out. When inspecting the contact points between the brushes and the slip rings, important differences were detected between rotor phases; an abnormal temperature was detected in the central ring ( 20-30 ${ }^{\circ} \mathrm{C}$ higher than the other two rings) reaching values above 80 ${ }^{\circ} \mathrm{C}$. This is illustrated in Fig. 13 that shows the infrared image indicating the defective contact in the central phase. Fig. 14 shows the equivalent infrared image for a similar motor operating in the same plant and driving an analogue mill; the infrared images show significantly lower temperature differences between phases (maximum differences of $2-3^{\circ} \mathrm{C}$ ).

Finally, the brushes system was replaced and the contact with the slip rings enhanced; consequently, a more uniform temperature distribution was obtained in later measurements. The proposed five-evidence-based method was applied again, obtaining significantly lower current signatures than before replacing the brushes. 
In summary, thanks to the proposed method, the presence of the rotor winding symmetry was successfully detected in the motor; this fact enabled to confirm the presence of the fault and forced the decision of using additional tools to locate the exact point where the asymmetry was caused, being successfully repaired and preventing an eventual forced motor outage.

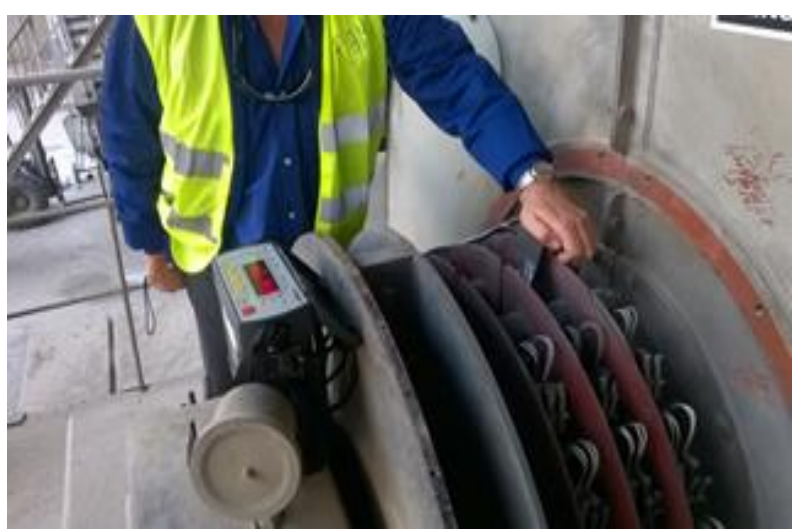

Fig. 12 Measurement of the resistive unbalance in the rotor winding with a digital microhmeter.

\section{CONCLUSIONS}

This paper proposes the use of current monitoring to detect rotor asymmetries in WRIM. The analysis of currents may provide interesting advantages in comparison with monitoring other quantities of the machine. A robust methodology based on five different fault evidences has been proposed. Four of these evidences rely on the analysis of stator currents (the registration of a single phase current is enough); two of them are based on detecting well-known families of fault harmonics appearing in the FFT spectrum of the steady-state current (MCSA) while the other two indicators rely on the analysis of the startup current via continuous transforms (evidence 3 ) and discrete (evidence 4).

The fifth evidence is based on the analysis of the rotor currents (when accessible) and the computation of the rotor currents space vector spectrum.

The proposed method has been tested on laboratory machines with diverse levels of artificially forced rotor winding asymmetry. Moreover, it has been validated in a real large field motor where the application of the method enabled to detect a significant level of rotor winding asymmetry, the origin of which was later localized by means of complementary tools.

Among the potential advantages of the proposed approach, we can find:

- High reliability, since the method relies on up to five different evidences of the presence of the fault. In this way, eventual false indications provided by a single technique are avoided.

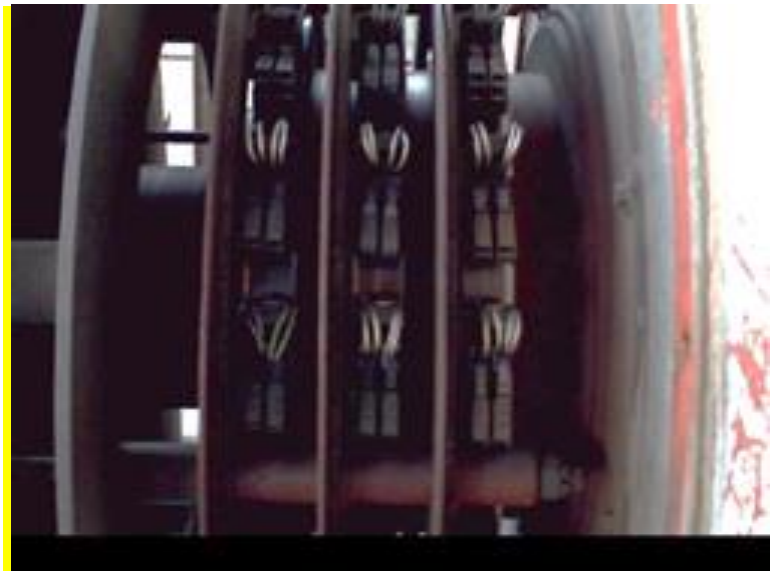

(a)

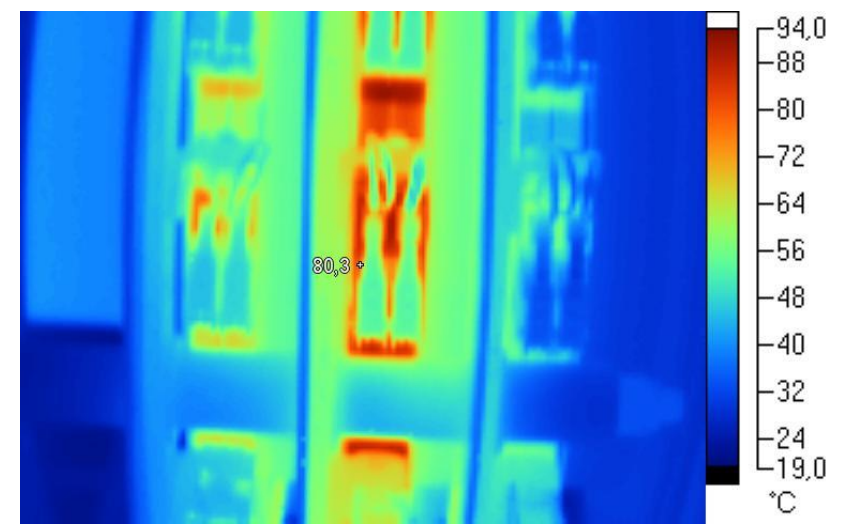

(b)

Fig. 13 (a) Slip-rings/brushes system and (b) thermographic inspection confirming the presence of the asymmetry in the rotor circuit

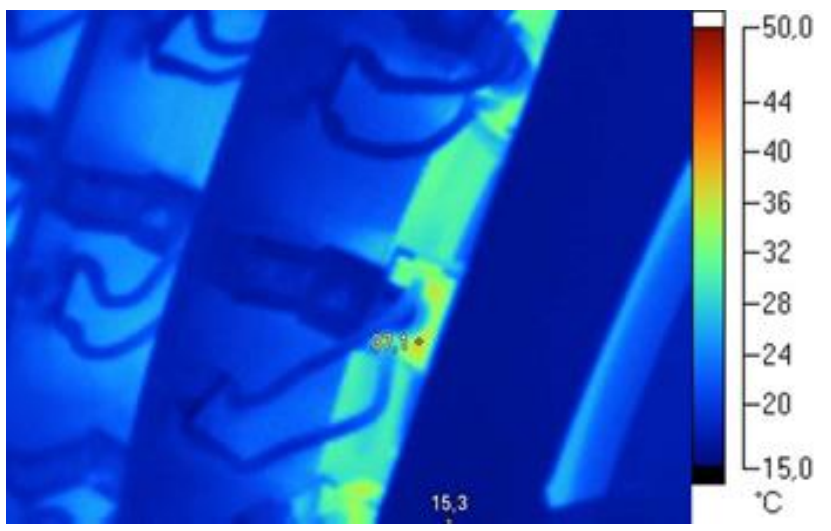

Fig. 14 Thermographic inspection of a similar motor with healthy slipring/brushes system.

- The method enables to obtain useful information to locate the origin of the fault; depending on if the fault components are present in steady-state and/or startup, some parts of the rotor circuit can be discarded as sources of the asymmetry (e.g. the starting rheostat can 
be discarded in the case that the fault signatures are present in steady-state).

- Versatility; the method can still be applied in case any quantity is not available (e.g. if rotor currents are not easily accessible, we still have four indicators based on the analysis of stator currents).

- Remote monitoring, non-invasive nature, simple application; the method has all the potential advantages derived from electrical monitoring, since it only relies on the analysis of machine currents.

The proposed reliable method is potentially applicable in a wide number of large WRIM operating in different applications such as mills, cranes, pumps, fans, blowers, conveyors, chippers or hoists. Most of these motors are large machine where the implications of excessive levels of rotor winding asymmetry may lead not only to a reduction in the motor efficiency or abnormal stresses on its components provoked by unbalanced rotor currents but also to catastrophic failures caused, for instance, by excessive heat dissipations due to defective connections in specific points of the rotor winding, as it was shown in the industrial example presented in this work

\section{ACKNOWLEDGMENTS}

This work was supported in part by the Spanish 'Ministerio de Economía y Competitividad' (MINECO) and FEDER program in the framework of the 'Proyectos I+D del Subprograma de Generación de Conocimiento, Programa Estatal de Fomento de la Investigación Científica y Técnica de Excelencia' (ref: DPI2014-52842-P)."

\section{REFERENCES}

[1] S.B. Lee, E. Wiedenbrug., K. Younsi, "ECCE 2013 Tutorial: Testing and Diagnostics of Induction Machines in an Industrial Environment", presented at ECCE 2013, Denver, CO, USA, Sep 2013.

[2] Jose A . Antonino-Daviu, A.Quijano-Lopez, V. Fuster-Roig and C. Nevot, "Case Stories on Induction Motors Fault Diagnosis based on Current Analysis", in Proc. of PCIC Europe 2016, Berlin, Germany, June 2016, pp. 115-123.

[3] S.B. Lee, "SDEMPED 2013 Tutorial: Testing and Monitoring of Medium/High Voltage Induction Machines", presented at the SDEMPED 2013, Valencia, SPAIN, Sep. 2013.

[4] W.T. Thomson, M. Fenger, "Current signature analysis to detect induction motor faults" IEEE Industry Applications Magazine, July/August 2001, pp. 26-34.

[5] A. Bellini, et al., "On-field experience with on-line diagnosis of large induction motors cage failures using MCSA," IEEE Trans. on Ind. Appl., pp. 1045-1053, vol. 38, no. 4, July/Aug. 2002.

[6] I.M. Culbert, and W. Rhodes, "Using current signature analysis technology to reliably detect cage winding defects in squirrel-cage induction motors," IEEE Trans. on Ind. Appl., vol. 43, no.2, pp.422-428, Mar./Apr. 2007

[7] Jose A. Antonino Daviu, Joan Pons-Llinares and Sang Bin Lee, "Advanced Rotor Fault Assessment for High Voltage Induction Motors via Continuous Transforms" in Proc. of PCIC Europe, London, U.K, June 2015, pp. 57-63.

[8] J. A. Antonino-Daviu, M. Riera-Guasp, J. R. Folch, and M. Pilar Molina Palomares, "Validation of a new method for the diagnosis of rotor bar failures via wavelet transform in industrial induction machines," IEEE Trans. Ind. Appl., vol. 42, pp. 990-996, 2006.

[9] S. Lee, J. Hong, S. B. Lee, E. J. Wiedenbrug, M. Teska and H. Kim, "Evaluation of the Influence of Rotor Axial Air Ducts on Condition
Monitoring of Induction Motors," IEEE Transactions on Industry Applications, vol. 49, no. 5, pp. 2024-2033, Sept.-Oct. 2013.

[10] S. Shin, J. Kim, S. B. Lee, C. Lim and E. J. Wiedenbrug, "Evaluation of the Influence of Rotor Magnetic Anisotropy on Condition Monitoring of Two-Pole Induction Motors," IEEE Transactions on Industry Applications, vol. 51, no. 4, pp. 2896-2904, July-Aug. 2015.

[11] J. Park, B. Kim, J. Yang, K. Lee, S.B. Lee, E.J. Wiedenbrug, M. Teska, and S. Han, "Evaluation of the Detectability of Broken Rotor Bars for Double Squirrel Cage Rotor Induction Motors," in proc. of the IEEE ECCE, pp. 2493-2500, Sept. 2010.

[12] J. A. Antonino-Daviu, V. Climente-Alarcón, J. Pons-Llinares and E. Wiedenbrug, "Advanced rotor assessment of motors operating under variable load conditions in mining facilities," in Proc. of the 2014 IEEE Energy Conversion Congress and Exposition (ECCE), Pittsburgh, PA, 2014, pp. 617-621.

[13] Jose Antonino-Daviu, Martin Riera-Guasp, Joan Pons-Llinares, Jongbin Park, Sang Bin Lee, Jiyoon Yoo and Christian Kral, "Detection of Broken Outer Cage Bars for Double Cage Induction Motors under the Startup Transient", IEEE Transactions on Industry Applications, vol. 48, no.5, pp. 1539-1548, Sept-Oct. 2012.

[14] C. Yang, T-J. Kang, D. Hyun, S. Lee, J. Antonino-Daviu, J. PonsLlinares, "Reliable Detection of Induction Motor Rotor Faults Under the Rotor Axial Air Duct Influence," IEEE Trans. Ind. Appl., vol. 50, no. 4, pp. 2493-2502, Jul.-Aug. 2014.

[15] J. A. Antonino-Daviu, S. B. Lee and E. Wiedenbrug, "Reliable detection of rotor bar failures in induction motors operating in petrochemical plants," in Proc. of the 2014 Petroleum and Chemical Industry Conference Europe (PCIC Europe), Amsterdam, 2014, pp.1-9.

[16] J. A. Antonino-Daviu, J. Pons-Llinares, Sungsik Shin, Kun Wang Lee and Sang Bin Lee, "Reliable detection of induction motor rotor faults under the influence of rotor core magnetic anisotropy," in Proc.of the 2015 IEEE 10th International Symposium on Diagnostics for Electrical Machines, Power Electronics and Drives (SDEMPED), Guarda, 2015, pp. $14-21$

[17] A. Stefani, A. Yazidi, C. Rossi, F. Filippetti, D. Casadei and G. A. Capolino, "Doubly Fed Induction Machines Diagnosis Based on Signature Analysis of Rotor Modulating Signals," in IEEE Transactions on Industry Applications, vol. 44, no. 6, pp. 1711-1721, Nov.-dec. 2008.

[18] Y. Gritli, L. Zarri, C. Rossi, F. Filippetti, G. A. Capolino and D. Casadei, "Advanced Diagnosis of Electrical Faults in Wound-Rotor Induction Machines," in IEEE Transactions on Industrial Electronics, vol. 60, no. 9, pp. 4012-4024, Sept. 2013.

[19] Y. Gritli, C. Rossi, D. Casadei, F. Filippetti, A. Bellini and G. A. Capolino, "A new reliable fault index for rotor dissymmetry detection in wound-rotor induction machine," 2015 IEEE 10th International Symposium on Diagnostics for Electrical Machines, Power Electronics and Drives (SDEMPED), Guarda, 2015, pp. 468-473.

[20] Staff of Lab-Volt Ltd. "Three-phase wound rotor induction machines", Courseware sample, 2011. http://www.labvolt.com/downloads/download/86367_F0.pdf

[21] TECO-Westinghouse Motor Company. "Wound rotor motor technology", Wound rotor motor brochure, http://www.tecowestinghouse.com/PDF/woundrotor.pdf

[22] Manés F. Cabanas, Manuel G. Melero, Gonzalo A. Orcajo, José M. Cano, Juan Solares, Maintenance and Diagnostic Techniques for Rotating Electric Machinery, Marcombo Boixareu, 1999.

[23] J. Pons-Llinares, J. A. Antonino-Daviu, M. Riera-Guasp, S. Bin Lee, T. J. Kang and C. Yang, "Advanced Induction Motor Rotor Fault Diagnosis Via Continuous and Discrete Time-Frequency Tools," IEEE Transactions on Industrial Electronics, vol. 62, no. 3, pp. 1791-1802, March 2015.

[24] J. Antonino-Daviu, V. Climente-Alarcon, I. Tsoumas, G. Georgoulas and R. B. Pérez, "Multi-harmonic tracking for diagnosis of rotor asymmetries in wound rotor induction motors," Industrial Electronics Society, IECON 2013 - 39th Annual Conference of the IEEE, Vienna, 2013, pp. 5555-5560.

[25] F.J. Vedreño Santos, "Diagnosis of electric induction machines in nonstationary regimes working in randomly changing conditions", $\mathrm{PhD}$ dissertation, Universitat Politecnica de Valencia, Valencia, Spain, 2013.

[26] L. Cohen. Time-Frequency Analysis Prentice-Hall, Englewood Cliffs, NJ, 1995. 
[27] J. Antonino-Daviu, A. Quijano-Lopez, V. Climente-Alarcon and C. Garín-Abellán, "Robust Detection of Rotor Winding Asymmetries in Wound Rotor Induction Motors via Integral Current Analysis", presented at 2016 IEEE Energy Conversion Congress and Exposition (ECCE 2016), Milwaukee, WI, USA, Sep.2016.

[28] H. Kim, S. B. Lee, S. Park, S. H. Kia and G. A. Capolino, "Reliable Detection of Rotor Faults Under the Influence of Low-Frequency Load Torque Oscillations for Applications With Speed Reduction Couplings," in IEEE Transactions on Industry Applications, vol. 52, no. 2, pp. 14601468, March-April 2016.

[29] N. Sarma, Q. Li, S. Djurovic, A. C. Smith and S. M. Rowland, "Analysis of a wound rotor induction machine low frequency vibroacoustic emissions under stator winding fault conditions," 8th IET International Conference on Power Electronics, Machines and Drives (PEMD 2016), Glasgow, 2016, pp. 1-6.

[30] S. Djurović, D. Vilchis-Rodriguez and A. C. Smith, "Vibration monitoring for wound rotor induction machine winding fault detection," 2012 XXth International Conference on Electrical Machines, Marseille, 2012, pp. 1906-1912.

[31] J. Faiz, M. Keravand and M. Ghasemi-Bijan, "Impact of rotor inter-turn short circuit fault upon performance of a wound rotor induction motor," 2015 Intl Aegean Conference on Electrical Machines \& Power Electronics (ACEMP), 2015 Intl Conference on Optimization of Electrical \& Electronic Equipment (OPTIM) \& 2015 Intl Symposium on Advanced Electromechanical Motion Systems (ELECTROMOTION), Side, 2015, pp. 681-686.

[32] D. E. Khodja and B. Chetate, "Torque based selection of ANN for fault diagnosis of wound rotor asynchronous motor-converter association,"
National Conference on Electrical, Electronics and Computer Engineering, Bursa, 2010, pp. 339-343.

[33] F. Vedreño-Santos, M. Riera-Guasp, H. Henao, M. Pineda-Sánchez and R. Puche-Panadero, "Diagnosis of Rotor and Stator Asymmetries in Wound-Rotor Induction Machines Under Nonstationary Operation Through the Instantaneous Frequency," in IEEE Transactions on Industrial Electronics, vol. 61, no. 9, pp. 4947-4959, Sept. 2014.

[34] Y. Gritli, C. Rossi, D. Casadei and F. Filippetti, "Square current spacevector signature analysis for rotor fault detection in wound-rotor induction machine," 2016 XXII International Conference on Electrical Machines (ICEM), Lausanne, 2016, pp. 2894-2898.

[35] S. H. Kia, H. Henao and G. A. Capolino, "Windings monitoring of wound rotor induction machines under fluctuating load conditions," IECON 2011 - 37th Annual Conference of the IEEE Industrial Electronics Society, Melbourne, VIC, 2011, pp. 3459-3465.

[36] M. Zaggout, P. Tavner, C. Crabtree and L. Ran, "Detection of rotor electrical asymmetry in wind turbine doubly-fed induction generators," in IET Renewable Power Generation, vol. 8, no. 8, pp. 878-886, 11 2014

[37] V. Climente-Alarcon, M. Riera-Guasp, J. Antonino-Daviu, J. RogerFolch and F. Vedreño-Santos, "Diagnosis of rotor asymmetries in wound rotor induction generators operating under varying load conditions via the Wigner-Ville Distribution," International Symposium on Power Electronics Power Electronics, Electrical Drives, Automation and Motion, Sorrento, 2012, pp. 1378-1383.

[38] W. Qiao and D. Lu, "A Survey on Wind Turbine Condition Monitoring and Fault Diagnosis-Part I: Components and Subsystems," in IEEE Transactions on Industrial Electronics, vol. 62, no. 10, pp. 6536-6545, Oct. 2015. 